\title{
Answers to paediatric crossword puzzle 33
}

Manouri P Senanayake ${ }^{1,2}$, A S Athapathu ${ }^{2}$

Sri Lanka Journal of Child Health, 2017; 46(1): 96

DOI: http://dx.doi.org/10.4038/sljch.v46i1.8232

\begin{tabular}{|c|c|c|c|c|c|c|c|c|c|c|c|c|c|}
\hline & ${ }^{1} \mathrm{O}$ & & ${ }^{2} \mathrm{~S}$ & $\mathrm{~W}$ & ${ }^{3} \mathrm{~A}$ & $\mathrm{~L}$ & $\mathrm{~L}$ & $\mathrm{O}$ & $\mathrm{W}$ & & & ${ }^{4} \mathrm{H}$ & \\
\hline${ }^{5} \mathrm{O}$ & $\mathrm{B}$ & $\mathrm{S}$ & & & $\mathrm{U}$ & & & & & & & $\mathrm{Y}$ & \\
\hline & $\mathrm{J}$ & & & & $\mathrm{D}$ & & & ${ }^{6} \mathrm{P}$ & & ${ }^{7} \mathrm{H}$ & $\mathrm{I}$ & $\mathrm{G}$ & $\mathrm{H}$ \\
\hline & $\mathrm{C}$ & & & & $\mathrm{T}$ & & & ${ }^{9} \mathrm{O}$ & $\mathrm{Z}$ & $\mathrm{O}$ & $\mathrm{N}$ & $\mathrm{E}$ & \\
\hline & $\mathrm{T}$ & & & ${ }^{10} \mathrm{Z}$ & & & & $\mathrm{M}$ & & & & $\mathrm{N}$ & \\
\hline & $\mathrm{S}$ & & & $\mathrm{E}$ & & & & $\mathrm{P}$ & & & ${ }^{11} \mathrm{M}$ & $\mathrm{E}$ & $\mathrm{A}$ \\
\hline${ }^{12} \mathrm{~S}$ & & & & ${ }^{13} \mathrm{R}$ & $\mathrm{I}$ & $\mathrm{G}$ & $\mathrm{H}$ & $\mathrm{T}$ & $\mathrm{S}$ & & & & \\
\hline $\mathrm{E}$ & & & & $\mathrm{O}$ & & & & $\mathrm{I}$ & & ${ }^{14} \mathrm{~F}$ & ${ }^{15} \mathrm{M}$ & $\mathrm{I}$ & \\
\hline${ }^{16} \mathrm{~N}$ & $\mathrm{I}$ & ${ }^{17} \mathrm{C}$ & $\mathrm{E}$ & & & & & $\mathrm{N}$ & & & $\mathrm{Y}$ & & \\
\hline $\mathrm{S}$ & & $\mathrm{R}$ & & & ${ }^{18} \mathrm{~B}$ & $\mathrm{E}$ & $\mathrm{I}$ & $\mathrm{G}$ & $\mathrm{H}$ & $\mathrm{T}$ & $\mathrm{O}$ & $\mathrm{N}$ & \\
\hline $\mathrm{O}$ & & $\mathrm{A}$ & & & $\mathrm{U}$ & & & & & & $\mathrm{P}$ & & \\
\hline${ }^{19} \mathrm{R}$ & $\mathrm{E}$ & $\mathrm{B}$ & $\mathrm{O}$ & $\mathrm{U}$ & $\mathrm{N}$ & $\mathrm{D}$ & & & & ${ }^{20} \mathrm{~L}$ & $\mathrm{I}$ & $\mathrm{C}$ & $\mathrm{K}$ \\
\hline $\mathrm{Y}$ & & & & & & & & & & & $\mathrm{A}$ & & \\
\hline
\end{tabular}

${ }^{1}$ Department of Paediatrics, Faculty of Medicine, Colombo

${ }^{2}$ University Paediatric Unit, Lady Ridgeway Hospital for Children, Colombo 